Journal für Mobilität und Verkehr

ISSN 2628-4154

www.dvwg.de

\title{
Die Aneignung neuer Mobilitätspraktiken. Eine qualitative empirische Analyse mit Nutzer:innen von Elektro-Motorroller-Sharing in München
}

\author{
Dr. Jessica Le Bris, Julia Korsten, Verena Prediger, Dr. Alexandra Bensler \\ Siehe AutorInnenangaben
}

\section{Abstract}

Seit einiger Zeit erweitern Elektro-Motoroller-Sharing-(EMS)-Systeme das Angebot innerstädtischer Fortbewegungsmöglichkeiten. Der Artikel gibt Einblicke in die Herausbildung der damit einhergehenden neuen EMS-Mobilitätspraktik. Auf qualitativen empirischen Analysen basierend, werden die vielfältigen Merkmale der Praktik deutlich. Dabei zeigen die Daten: Mobilität und die EMS-Nutzung sind mehr als nur reine Fortbewegung! Qualitative Faktoren wie Emotionen, persönliche Wahrnehmungen und soziale Prozesse sind wesentliche Elemente der EMS Praktik.

Schlagwörter/Keywords:

Elektro-Motorroller-Sharing, E-Scooter, Mobilitätspraktiken, Aneignung, qualitative empirische Analyse, interpretative Sozialforschung

\section{Einleitung}

Immer mehr Städte leiden unter den negativen Konsequenzen eines autozentrierten Verkehrssystems. Verkehrsstau, steigende Emissionen und Lärm sowie steigender Parkplatzdruck sind Entwicklungen, die sich zunehmend verschärfen. Zugleich wird der Markt an Mobilitätslösungen und -anbietern immer vielfältiger und differenzierter. Während sich Car-Sharing bereits vielerorts etabliert hat, stellen SharingSysteme, deren Flotte rein aus Elektro-Motorrollern besteht, ein relativ neues Mobilitätsangebot auf dem europäischen Markt dar. Als Teil eines integrierten Mobilitätssystems bieten sie die Chance, das Mobilitätsangebot einer Stadt vielfältiger zu machen, neue Nutzer:innengruppen zu erschließen, den motorisierten Individualverkehr (MIV) zu reduzieren und versprechen, zu einer saubereren, flexibleren und platzsparenden Mobilität beizutragen.

Der Artikel beruht auf einer übergeordneten Studie zu Elektro-Motorroller-Sharing (EMS) ${ }^{1}$, die im Rahmen des EU-För-

\footnotetext{
1 Da es bis dato noch keine differenzierte und einheitliche Begriffsdefinition für Leihsysteme von E-Rollern gibt, wird im Folgenden der Begriff Elektro-Motoroller-Sharing verwendet, kurz EMS. Teilweise wird für Elektro-Motoroller auch der Terminus E-Scooter verwendet, der jedoch häufig auch für E-Tretroller steht. Diese sind nicht Gegenstand der vorliegenden Forschung.
}

derprojekts (Horizon 2020) Civitas Eccentric in München durchgeführt wurde. Untersucht wurden Fragestellungen zu den Bereichen Nutzer:innengruppen, Nutzungsverhalten, Verlagerungspotenziale und Handlungsbedarfe zur Steuerung der verkehrlichen Wirkungseffekte. Im Zuge der Studie wurden Fahrtdaten ausgewertet, eine Online-Umfrage mit EMS-Nutzenden sowie zwölf vertiefende qualitative Interviews durchgeführt. Fokus dieses Artikels ist die Vorstellung ausgewählter Ergebnisse der qualitativen Teilstudie, die mit Kund:innen von EMS durchgeführt wurde. Dabei steht die individuelle Wahrnehmung der EMS-Praktik durch die Nutzer:innen selbst im Vordergrund: Analysiert werden die Aneignung und Nutzungspraxis des neuen Angebots. Im Zuge dessen soll das Nutzerhandeln im Umgang mit EMS als Teil der neuen Mobilitätspraktik nachvollzogen werden. Das Konzept von Mobilitätspraktiken wurde u. a. gewählt, da sich in der Analyse der Daten die Herausbildung von Emotionen, Sinnempfindungen und individuellen Bedeutungszuschreibungen während der Nutzung von EMS in besonderer Weise herauskristallisiert hat - der praxistheoretische Ansatz zu sozialen Praktiken und das Konzept der Aneignung messen diesen Elementen einen wesentlichen Stellenwert bei. 


\section{Stand der Forschung zu Elektro-Motorroller-Sharing (EMS)}

Im Bereich Sharing gibt es bereits eine Reihe von Studien, die das Thema Nutzungsverhalten und Akzeptanz für die Systeme Car-Sharing, Bike-Sharing oder mittlerweile auch Tretroller-Sharing in den Blick nehmen. ${ }^{2}$ Die Erforschung von EMS steht dagegen noch ganz am Anfang. Die wenigen vorhandenen Analysen zu EMS beruhen überwiegend auf quantitativen Forschungsdesigns. ${ }^{3}$ In einer Pilotstudie in München wird zwar nicht direkt ein bestehendes Sharing-System analysiert, doch es werden Fragen zur Nutzerakzeptanz und Einstellungen gegenüber E-Motorrollern aufgegriffen. Die Daten aus der Vorher-Nachher-Befragung von Testnutzenden zeigen, dass das Sicherheitsempfinden im Straßenverkehr, Wetter und Transportkapazitäten die Nutzungsakzeptanz beeinflussen. Dazu wird festgestellt, dass die Bereitstellung von Rollern im Rahmen einer Testperiode Perzeptionen und Einstellungen gegenüber dem neuen Fahrzeugtyp verändert hat. So wurde z.B. die Wahrnehmung von E-Rollern im Straßenbild geschärft und die Angst vor mangelnder Ladeinfrastruktur nahm ab. Ein Bezug zum theoretischen Konzept der Aneignung neuer Technologien wird jedoch nicht hergestellt. Vertiefende Forschungsstudien, die über eine Bewertung vorgegebener Kategorien hinausgehend individuelle Perspektiven von E-Roller-Nutzenden auf Basis qualitativer Analysen untersuchen, konnten nicht identifiziert werden.

In der bereits erwähnten, eigens durchgeführten und übergeordneten Studie zum Nutzungsverhalten von EMS wurden ebenfalls Fahrdaten des EMS analysiert (Erhebungszeitraum 2019) ${ }^{4}$. Zur Einbettung der qualitativen Daten sind hier die wichtigsten Ergebnisse kurz zusammengefasst: durchschnittliche Fahrtdauer der Elektro-Motorroller 13 Minuten, rund 10\% der Fahrten kürzer als ein Kilometer, durchschnittliche Fahrtlänge pro Tag 4,21 km, nutzungsstärkste Wochentage Freitag und Samstag, Spitzen der zeitlichen Verteilung Montag bis Freitag jeweils um 8 Uhr und 18 Uhr, Mehrheit der Fahrten im Juni und Juli (in Korrelation mit der durch-

\footnotetext{
2 (vgl. Aguilera-García et al. 2021)

3 So wurden bspw. in Spanien in einigen Städten Erhebungen durchgeführt, die entweder auf GPS-Fahrtdaten oder quantitativen Online-Befragungen basieren (Pérez-Fernández und García-Palomares 2021; AguileraGarcía et al. 2019; Aguilera-García et al. 2021)

Auch in Stuttgart wurde eine Studie zur Analyse eines EMS Systems veröffentlicht, im Fokus steht die Clusterung von Konsumentengruppen (Degele et al. 2018). Eine weitere Studie in München beruht auf einen Feldtest, in dem Mobilitätstagebücher von 35 Probanden ausgewertet sowie eine quantitative Vorher-Nachher-Befragung der Probanden durchgeführt wurde (vgl. Hardt und Bogenberger 2019). Dabei unterscheidet sich jedoch der konkrete Forschungsgegenstand der Pilotstudie zu dem des vorliegenden Artikels: Während dort Proband:innen ein Fahrzeug (Elektro-Motorroller) zum privat organisierten Teilen zur Verfügung gestellt wurde, so beruhen die hier vorgestellten Ergebnisse auf den Analysen von Kundinnen eines bereits bestehenden Free-Floating-Systems inklusive One-Way Nutzungen.

4 Bis dato liegen diese Ergebnisse nur im Rahmen eines Forschungsberichts vor.
}

schnittlichen Monats-Temperatur).

Darüber hinaus zeigen die Ergebnisse der quantitativen Befragung (553 Fälle): bzgl. Nutzungshäufigkeit von EMS: 8\% nutzen EMS fast nie (und haben sich nur registriert), 34\% nutzen EMS weniger als einmal im Monat, 45\% zwischen ein bis drei Tagen im Monat und $12 \%$ zwischen ein bis drei Tage die Woche und 1\% täglich; bei Substitutionseffekten bzgl. der Verkehrsleistung lässt sich feststellen: gefahrene Kilometer, die substituiert wurden, betragen im Sample 60\% MIV-Kilometer; 32\% ÖPNV-Kilometer, 6\% Rad-Kilometer und 2\% Fuß-Kilometer; bei Substitutionseffekte bzgl. des Verkehrsaufkommens zeigt sich: am häufigsten wird der ÖPNV (26\%) ersetzt, gefolgt vom Rad und zu Fuß gehen (je 19\%). Fahrten mit dem MIV werden zu 12\% substituiert; 4\% sind Fahrten „des Fahrens wegen“, d.h. diese wurden neu generiert; wichtigste Nutzungsmotivationen sind Spaß (59\%), keinen Parkplatz suchen müssen (46\%) und Schnelligkeit (38\%).

\section{Das Forschungsdesign der qualitativen Teilstudie}

Das untersuchte EMS-System umfasst ca. 45.000 Kund:innen mit rund 400 Rollern im Stadtgebiet von München und wird als free-floating-Dienstleistung betrieben (Stand Juli 2020). Die übergreifende Studie zu EMS basiert auf einem MixedMethods-Forschungsansatz. Im Fokus dieses Artikels stehen die qualitativen Analysen im Rahmen der Nutzer:inneninterviews, um die Herausbildung der neuen Mobilitätspraktik als neues Mobilitätsangebot zu verstehen.

\subsection{Leitende Forschungsfragen}

Leitende Forschungsfrage für das Gesamtprojekt war, inwieweit EMS zu mehr nachhaltiger Mobilität in europäischen Städten beiträgt. Um dieses Potenzial zu eruieren und ggf. Ansatzpunkte zur Optimierung zu entwickeln, ist es wichtig, zunächst das bestehende Nutzungsverhalten zu analysieren und relevante Handlungsmuster zu identifizieren. Hier bietet der Blick darauf, wie sich eine neue Mobilitätspraktik aus der persönlichen Perspektive der Nutzenden selbst entwickelt, wertvolle Einsichten, um individuelle Handlungslogiken nachzuvollziehen. Für die vorliegende qualitative Teilstudie standen folgende Leitfragen im Vordergrund: Wie kam es zur Nutzung von EMS? Was passiert während der Nutzung von EMS und wie hat sich die Nutzungspraxis im Laufe der Zeit verändert? Welche Elemente prägen heute die Nutzung? Letztlich basiert das vorliegende Paper auf der (praxistheoretischen) Frage: Wie kommt es zur Herausbildung der EMSPraktik und wodurch ist die EMS-Praktik charakterisiert? 
Abbildung 1: Modell der individuellen Mobilitätskarrieren (MoKa) inkl. der Aneignung (eigene Darstellung modifiziert auf Basis von Le Bris 2015)

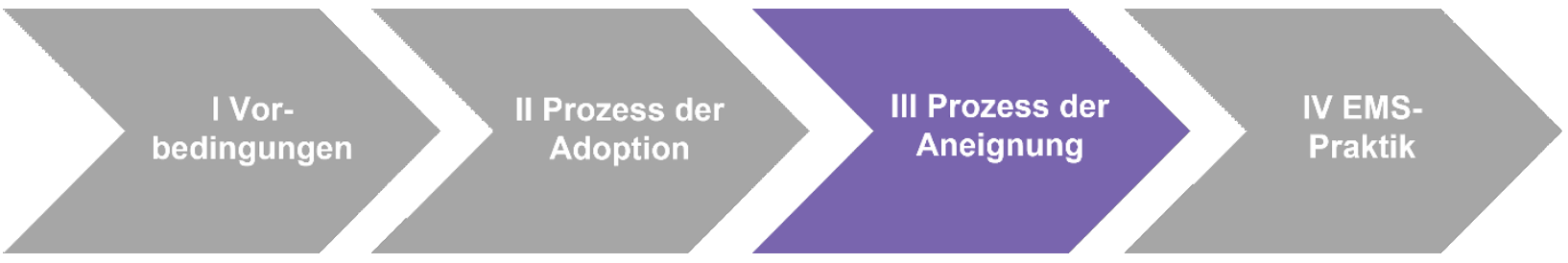

\subsection{Datengrundlage und Auswertungsmethodik}

Neben der Analyse getrackter (anonymisierter) Fahrdatensätze ${ }^{5}$ erfolgte im September 2019 eine quantitative Nutzer:innenumfrage unter den EMS-Kund:innen. Die Online-Befragung enthielt Fragen zu Mobilitätsmustern und Substitutionseffekten sowie zur Nutzungsmotivation von EMS. Die Stichprobe der quantitativen Erhebung bestand aus 553 vollständigen Fragebögen. Dabei konnten Kontaktdaten angegeben werden, sofern die Bereitschaft bestand, an weiterführenden Interviews teilzunehmen. Insgesamt konnten über diese Akquisemöglichkeit 12 leitfadengestützte Interviews im Erhebungsraum München im Zeitraum April/Mai 2020 durchgeführt werden. Im Vordergrund standen Fragen zu den Erfahrungen und persönlichen Wahrnehmungen im Umgang mit dem System. Ausgewertet wurden die Interviews in Anlehnung an die Grounded Theory Methodologie ${ }^{6}$. Als heuristischer Rahmen dienten Theorien zur Herausbildung von Mobilitätspraktiken und zum Prozess von Technologieaneignung.

\subsection{Theoretischer Kontext zur Herausbildung von Mobili- tätspraktiken und Aneignungsprozessen}

Den theoretischen Rahmen der Analyse bilden das Modell der individuellen Mobilitätspraxis (MiMP) ${ }^{7}$ sowie das Modell der Mobilitätskarrieren (MoKa). ${ }^{8}$ Das MiMP beschreibt die relevanten Elemente einer Praktik und das MoKa die Phasen, über die sich die individuelle Mobilitätspraktik herausbildet. In den Modellen wird sowohl eine handlungstheoretische als auch praxistheoretische Perspektive integriert. Elemente bzw. Komponenten einer Praktik sind die Technologie selbst (Artefakte, Mobilitätsprodukte), das Wissen und Kenntnisse (Fähigkeiten) zur Nutzung von EMS sowie die Sinngebung oder Sinnstiftung der Praktik inklusive emotionaler Verknüpfungen. Ebenso wichtige Elemente sind die physisch-materielle Umwelt (z. B. der Raum inkl. Topographie, Siedlungs-/ Verkehrsstrukturen, Klima), soziale Lebenskontexte und auch die Verbindung zu anderen bestehenden Praktiken wie

\footnotetext{
5 Als Datenquellen standen (ca. 490.000 automatisch getrackte Fahrtdatensätze der Jahre 2018/2019) zur Verfügung.

6 (vgl. Mey und Mruck 2011)

7 (vgl. Le Bris 2015)

8 (vgl. Le Bris 2015)
}

die Praktiken des Autofahrens, Fahrradfahrens, Sportpraktiken, Konsumpraktiken usw. Darüber hinaus spielen tieferliegende Gewohnheiten (Lebensprägungen) sowie (erlernte) Alltagsroutinen eine tragende Rolle. ${ }^{9}$ Der Ansatz des MiMP geht davon aus, dass soziale Prozesse inkl. Praktiken nur durch eine ganzheitliche Perspektive und Integration aller relevanten Elemente bzw. Komponenten betrachtet werden können.

Die Aneignung neuer Technologien lässt sich als einen Abschnitt des MoKas systematisieren (vgl. Abbildung 1). Das MoKa umfasst insgesamt vier Phasen: Die (I) Vorbedingungen prägen die Herausbildung der Praktik durch habituelle Skriptformierungen, die u. a. auf Mobilitätsbiographien, Erfahrungs- und Sozialisierungskontexte, Orientierungen bzw. Einstellungen zurückgehen. Während des (II) Adoptionsprozess der neuen Technologie ${ }^{10}$ wird das neue Mobilitätsangebot wahrgenommen, worauf die Entscheidungs- und Abwägungsphasen folgen und schließlich die Anmeldung zum Sharing-Dienst. Die Phase umfasst zudem Schlüsselereignisse im Sinne von Adoptionsfenstern oder auch Adoptionsrisiken. Darauf folgt die (III) Phase der Aneignung selbst. ${ }^{11}$ Hier erfolgt die erste Nutzung der neuen Technologie und das Erlernen des Umgangs mit dem neuen Objekt.

Während der Aneignungsphase stehen Akteur:in und Artefakt, Mensch und Maschine bzw. Nutzer:in und Technologie in einem dynamischen Wirkungsverhältnis. ${ }^{12}$ Praxistheoretisch betrachtet besitzen und entfalten Artefakte wie der Elektro-Motoroller und das Sharing-System als Dienstleistungsangebot eine eigene Wirkkraft, die bspw. neue Handlungsweisen seitens der Nutzenden auslöst und neue Wahrnehmungen schafft. Umgekehrt modifiziert der/die Kunde:in ggf. aber auch im Laufe der Nutzung die Art und

\footnotetext{
9 Die genannten Elemente werden im MiMP in vier Komponentenbereiche zusammengefasst: Materialitäten, Wissen \& Sinn, Tun \& Sozialer Lebenskontext und habituelles Skript. Das MiMP stellt u.a. eine Erweiterung des Modells von Shove et al. (2012) dar, in dem Praktiken als Produkt von materials, meanings and competences verstanden werden.

10 (u.a. Rogers 2003)

11 (u.a. Degele 2002; Pinch und Bijker 1984; Oudshoorn und Pinch 2007) 12 Stark vereinfacht gesagt liegt z. B. bezogen auf die EMS Praktik ein Aneignungsprozess vor, wenn z. B. entweder die Technik etwas mit dem Menschen macht (und zum Beispiel Emotionen hervorruft), der Mensch sich bzw. sein Nutzungsverhalten an die technischen Gegebenheiten anpasst (und z. B. hygienische Maßnahmen bei der Kopfbedeckung trifft) oder aber auch die Technik selbst modifiziert (sofern möglich, wenn z. B. ein Roller im Privatbesitz vorhanden ist).
} 
Weise, wie mit dem Angebot konkret umgegangen wird. In der Phase der Aneignung wirken somatische Sinnwahrnehmungen, Affekte und Emotionen sowie körperliche Reaktionen während der Technologienutzung (Performanz der Praktik). Die Phase der Aneignung ist abgeschlossen, wenn sich Handlungsmuster verstetigt haben - ggf. wurden auch Routinen ausgebildet. In der letzten Phase haben sich individuelle Erfahrungsweisen, Bedeutungszuschreibungen und Handlungsmuster gebildet. Die neue Praktik ist als Teil der individuellen Mobilitätspraxis internalisiert. ${ }^{13}$

\section{Ergebnisse}

Im Laufe der Interviews konnte umfangreiches Datenmaterial zur Analyse der Mobilitätspraktiken von EMS-Kund:innen generiert werden. Die nachfolgenden Darstellungen fußen auf empirischen Ausschnitten, die die Herausbildung der EMS-Praktik bzw. ausgewählter Teilaspekte besonders charakterisieren. Die Struktur der Darstellungen orientiert sich an den vier Phasen des MoKas. Dort, wo Bezüge zu den Ergebnissen aus der quantitativen Befragung hergestellt werden können, erfolgt eine Verknüpfung über Fußnoten. Schwerpunkt ist jedoch eine differenzierte Erfassung der relevanten Hintergründe und individuellen Empfindungen, die über die explizit erfassbaren Kategorien des (quantitativ strukturierten) Fragebogens hinausgehen. ${ }^{14}$

\subsection{Vorbedingungen zur Herausbildung der EMS-Praktik}

Bei allen Interviewten lässt sich eine positive Prädisposition gegenüber Sharing-Angeboten erkennen. Angebotsformen, die eine Alternative zum MIV bieten, werden begrüßt (ql-02 Pos. 36) und eine Palette an Auswahlmöglichkeiten befürwortet. Der Nutzung des EMS-Systems ging häufig bereits die regelmäßige Nutzung von Car-Sharing-Angeboten voraus (ql-04 Pos.57, ql-08 Pos.5, 42, ql-11 Pos.18, 29) ${ }^{15}$. Bezüglich der E-Tretroller-Angebote herrschen verschiedene Meinungen vor: Teils gehört ihre Nutzung zum eigenen Mobilitätsrepertoire (ql-11 Pos.22, 56), teils werden sie explizit abgelehnt (ql-12 Pos.26, ql-07 Pos.10). Dem Fahrrad gegenüber lässt sich eine positive Orientierung feststellen (ql-06 Pos.11, Pos. 47, ql-12 Pos.7), es wird als das schnellste Verkehrsmittel innerhalb der Stadt empfunden. Auch der ÖPNV wird überwiegend positiv bewertet (ql-03 Pos.44, ql-07 Pos.21, ql-11 Pos.12-14) ${ }^{16}$, jedoch werden zugleich seine Limitierungen deutlich wahrgenommen. Nichtsdestotrotz gibt es auch Personen, die den ÖPNV als nicht akzeptabel empfinden (ql02 Pos. 22). Im Vergleich zum ÖPNV wird EMS eine größere Flexibilität zugesprochen (Tür-zu-Tür-Mobilität, Nutzungszeiten) (ql-02 Pos.32), was schließlich auch zur EMS-Nutzung motiviert. Im Interviewsample fällt auf, dass die Nutzung verschiedener Verkehrsmittel schon in den früheren Mobilitätsbiographien ein fester Bestandteil der Alltagspraxis war. Es wurde eine überwiegend multimodale Mobilitätspraxis ausgeübt. Auch wurden verschiedene Mobilitätsformen während einer Wegestrecke kombiniert (ql-05 Pos.30). Hierbei spielte der Umweltverbund eine besondere Rolle. ${ }^{17}$ Das Auto hat für viele innerhalb der Stadt eine untergeordnete Rolle und wird auch als unpraktisch bezeichnet (eingeschränkte Parkplatzsituation, dichter Verkehr, Stau). Eine spezifische Autoaffinität oder umgekehrt auch ideologisch begründete Aversion ließ sich in den Interviewdaten nicht feststellen, die Einstellung scheint eher pragmatisch orientiert (ql-09 Pos.34, ql-11 Pos.12-14, ql-12 Pos.7).

\subsection{Adoptionsprozess der EMS-Praktik}

Vorangegangene Erfahrungen, die zur Anmeldung für das EMS motiviert haben, waren u. a. starke Unzufriedenheit mit der bestehenden Autopraktik (qi-07 Pos.3) und der konkrete Wunsch, das Auto abzuschaffen. Aber auch negative Erfahrungen mit dem ÖPNV haben die Anmeldung für das neue Mobilitätsangebot vorbereitet (ql-07 Pos. 10), wenngleich dem ÖPNV eine hohe Bedeutung beigemessen wird. Ein Adoptionsfenster war in einem konkreten Fall der Ausfall der S-Bahn (ql-07 Pos. 10). In anderen Fällen wurde die bisherige Radpraktik als zu anstrengend empfunden (ql-08 Pos.7). Dazu wurde auch reine Neugier als Auslöser genannt (ql-09 Pos.32, ql-11 Pos. 58, ql-12 Pos.5) oder die positiven Erzählungen über das neue Angebot in der eigenen PeerGroup (ql-06 Pos. 38, 42). In einem weiteren Fall hat das erste konkrete Erleben, wie z. B. eine spontane Einladung einer Freundin für eine Mitfahrt auf dem Elektro-Motoroller, dazu geführt, die Technologie selbst nutzen zu wollen (ql-08 Pos. 7). Auch dies stellte einen auslösenden Schlüsselmoment dar. Ebenso hat das Angebot von Freiminuten den Schritt zur ersten Anmeldung unterstützt (ql-06 Pos. 38). Darüber hinaus hat sich gezeigt, dass die Sichtbarkeit und zunehmende Normalität von EMS im öffentlichen Raum zusätzlich die vorhandene Offenheit unterstützt haben, tatsächlich zur Anmeldung zu schreiten (ql-09 Pos.32).

\footnotetext{
13 (u.a. Silverstone und Hirsch 1992)

14 Die Analyse der Interviews gibt Aufschluss über die Relevanz und Vielfalt der wesentlichen Elemente und Ausprägungen. Quantitative Verteilungen sollen und können hier nicht überprüft werden. Aus den Daten können dagegen Tendenzen aufgestellt werden, die als Basis für weiterführende Hypothesen dienen.

15 Ergebnis der quantitativen Befragung: nur 12\% geben an, weder kommerziell noch privat ein Fahrzeug zu teilen.
}

16 Ergebnis der quantitativen Befragung: 41\% verfügen über eine ÖPNVMontas- oder Jahreskarte

17 Ergebnis der quantitativen Befragung: Der häufigste (fast) tägliche Bewegungsmodus der EMS-Nutzer:innen ist das zu Fuß gehen (67\%) gefolgt vom Radfahren (38\%), dem ÖPNV (37\%) und dem Auto (22\%); 61\% der Nutzer:innen nutzen täglich mind. zwei verschiedene Verkehrsmittel und mehr 


\subsection{Die Dimensionen der Aneignung der EMS-Praktik}

Während der Aneignungsphase stehen die Wechselwirkungen zwischen Technologie und Nutzenden im Vordergrund. Gemäß der theoretischen Fundierung erzielen Artefakte (also die Elektro-Motoroller und das SharingSystem) erst in der eigentlichen Nutzung bzw. im Vollzug der Praktik (Performanz) ihre individuelle Bedeutung. Das heißt, ursprüngliche Handlungsintentionen, die in der Regel rational begründet sind, werden im Verlauf der Aneignung eines Artefakts häufig modifiziert, transformiert und erweitert.

In den Daten konnten verschiedene Dimensionen identifiziert werden, in denen differenzierte Aneignungsprozesse stattfanden. In der grafischen Darstellung (Abbildung 2) sind die in den empirischen Daten identifizierten Dimensionen in ihrer Gesamtheit dargestellt: der Aufbau von Wissen bzw. Kompetenzen (kognitive Aneignung), die Ausbildung von Bedeutungszuschreibungen oder Wahrnehmung sinnstiftender Elemente (symbolische Aneignung), das Auslösen emotionaler Reaktionen oder der Aufbau emotionaler Verbindungen (emotionale Aneignung), körperliche Dynamiken, die in Wechselwirkung mit Gesundheit und dem Körpergefühl stehen (körperliche Aneignung), räumliche Wahrnehmungen oder Veränderung von Raummusters (räumliche Aneignung), die praktische Aneignung des EMS (z.B. Nutzungszwecke, Veränderungen der Nutzung im Zeitverlauf)

Abbildung 2: Die Dimensionen der Aneignung der EMS-Praktik (eigene Darstellung)

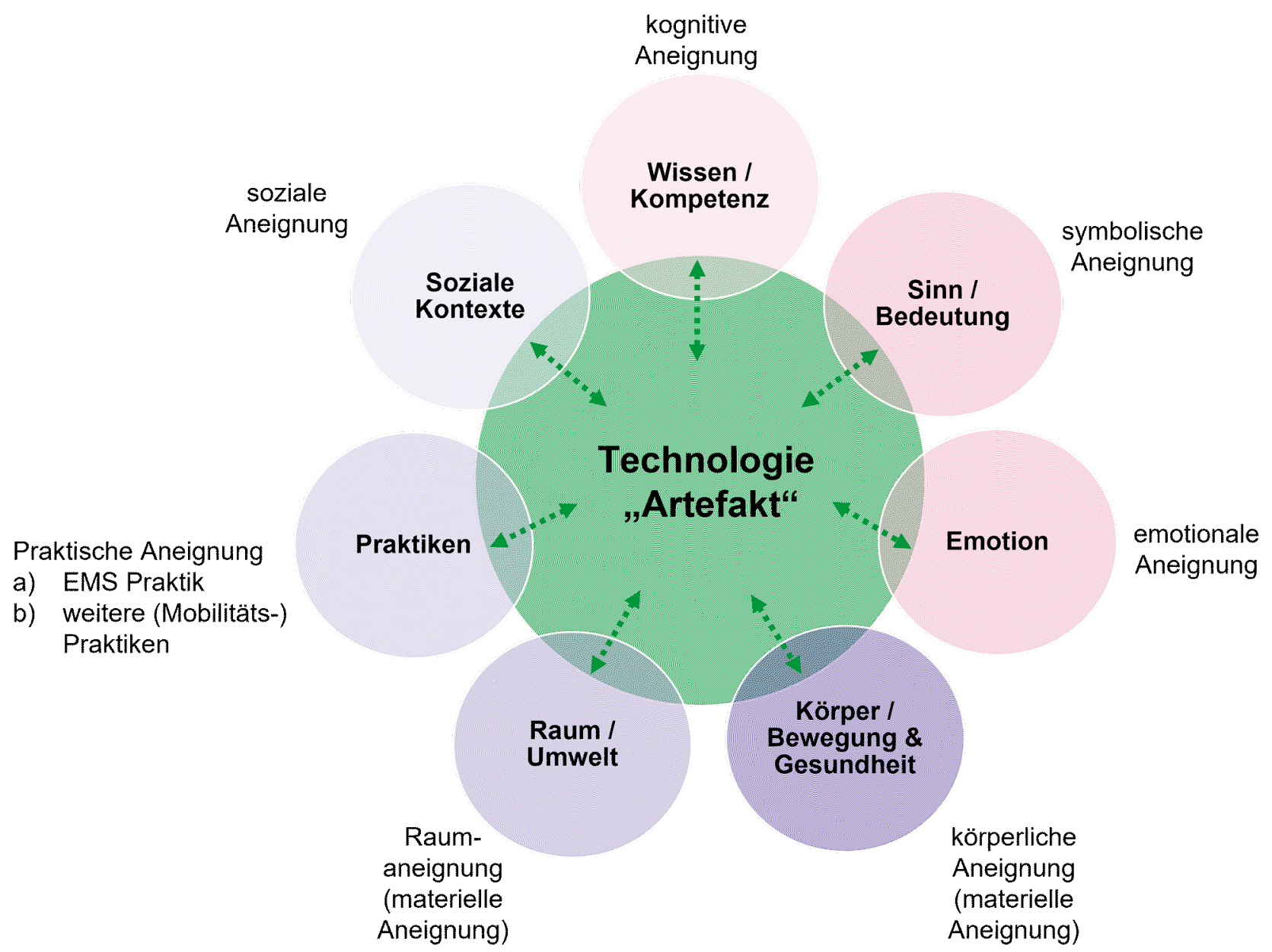

und Wechselwirkungen mit anderen Praktiken, wie z. B. Energienutzung sowie Prozesse, die in Verbindung mit dem sozialen Umfeld stehen (soziale Aneignung). ${ }^{18}$

Die empirischen Verknüpfungen zu den identifizierten Dynamiken werden nachfolgend je Dimension illustriert.

18 In den Theorien zu sozialen Praktiken werden Körper, Artefakte und die physisch-räumliche Umwelt auch zu Materie zusammengefasst, womit auch von materieller Aneignung gesprochen werden kann (Le Bris 2015).

\section{KOGNITIVE ANEIGNUNG}

Wenngleich Vorerfahrungen mit motorisierten Zweirädern vorhanden waren, wird berichtet, dass nach der ersten Anmeldung zunächst ein paar Test- bzw. Ausprobierfahrten mit der neuen Technologie unternommen wurden. Durch das Sichvertrautmachen und Üben (ql-03 Pos.27, ql-07 Pos.8) wurde das Wissen und die Kompetenz zum Handling aufge- 
baut. Dabei wurde auch ganz bewusst das Gefühl der Sicherheit überprüft, gestärkt (ql-08 Pos.19, ql-09 Pos.15) oder auch gleich zu Beginn der Nutzung bestätigt.

\section{SYMBOLISCHE ANEIGNUNG}

In einem Beispiel wird die Nutzung des EMS als Teil eines "Lebensgefühls" bezeichnet (ql-09 Pos.22) und berichtet, dass das EMS in München positiv konnotiert ist. „Also immer, wenn die Leute [ein EMS-Fahrzeug] sehen [...] finden die das geil und lächeln. [Der EMS-Anbieter] hat ein ziemlich gutes Image. Wenn du [das] fährst, hast du ein gutes Image, irgendwie." (ql-09 Pos.22) Das Beispiel zeigt die Bedeutung von Semantiken, die nichts direkt mit der Fortbewegung selbst zu tun haben. Der nicht-instrumentelle Mehrwert der Praktik äußert sich in der symbolischen Deutungszuschreibung durch das äußere Umfeld, aber auch in der eigenen Wahrnehmung. Das positive Image der Marke trägt hier zur positiven (Selbst-) Wahrnehmung des Nutzenden bei. ${ }^{19}$

\section{EMOTIONALE ANEIGNUNG}

Sich auf dem neuen Verkehrsmittel sicher zu fühlen, beeinflusst stark die persönliche Verkehrsmittelwahl. Wie sich in den Interviews zeigt, musste ein Sicherheitsgefühl teils zu Beginn erst (durch Üben) aufgebaut werden (ql-09 Pos.15) (vgl. kognitive Aneignung). Teils riefen die Elektro-Motorroller schon von Anfang an ein Sicherheitsgefühl hervor (ql-12 Pos.12, 04 Pos.45).

Darüber hinaus wird berichtet, dass das konkrete Ausüben der Praktik (also die Nutzung des Elektro-Motorrollers) ein Lächeln ins Gesicht ruft, auch wenn man vorher angespannt war (ql-10 Pos.10): „Und ich war dann glücklich [...] eine luftige Heimfahrt auf einem Elektroroller genießen zu können, das ist eigentlich eine sehr, sehr angenehme Situation dann gewesen. Hat meine Laune sofort verbessert." (ql-10 Pos. 6). In anderen Interviews wird erwähnt, wie schön es ist, den „Fahrtwind in den Haaren zu spüren“ (ql-06 Pos.17). Oder „Es ist alles offen und luftig und wenn es schön warm ist und der warme Wind fegt dir um die Ohren, dann ist es halt schön. Das macht Spaß." (ql-09 Pos.13) Wie die Beispiele zeigen, sind affektive Sinneswahrnehmungen während des Vollzugs der Praktik von großer Bedeutung. Dabei trägt die Praktik zur Schärfung der Sinne bei: „es ist halt immer wieder schön, dann mit offenen Augen zu seinem Termin zu fahren und zu genießen, wie schön es einfach ist." (ql-03 Pos.48, 49) Darüber hinaus wird die Praktik mit weiteren Assoziationen hinterlegt wie eine positive Verknüpfung mit den Jahreszeiten: „Aber generell verbinde ich einfach [das Produkt] mit lauen Sommernächten und schönen Sommertagen." (ql-08 Pos.25) Bei schlechten klimatischen Bedingungen dreht sich jedoch diese Wahrnehmung (zumindest für den Moment) ins Gegenteil: „nass werden, hab“ ich keine Lust. Oder auch

19 Inwiefern sich dies nur auf den spezifischen Anbieter bezieht oder auf EMS allgemein, bleibt an dieser Stelle offen. im Winter mit dem Fahrtwind, wird es immer kälter als es eigentlich ist." (ql-01 Pos.13) Für viele Nutzer:innen steht eine Fahrt mit dem Elektro-Motorroller jedoch unabhängig der aktuellen Wettersituation für Genuss, Freude, Spaß (ql03 Pos.48, 49). „Dieses Feeling ist schwer zu beschreiben." (ql-06 Pos.17).

\section{KÖRPERLICHE ANEIGNUNG (materielle Aneignung)}

Der Prozess der körperlichen Aneignung bezieht sich auf haptische Empfindungen bzw. körperliches Befinden oder gesundheitliche Aspekte. In einem Interview wird z. B. berichtet, dass die hygienischen Empfindungen bei der Nutzung des zur Verfügung gestellten Helms unter Umständen auch von der Nutzung abhalten. ${ }^{20}$ Jedoch wurde direkt eine Strategie entwickelt, wie mit dem persönlich empfundenen Problem umgegangen werden kann: „Und ich hab' da auch immer eine Kopfhaube. Einen Buff-Schal, wo ich dann halt einmal um meinen Kopf wickle. Damit ich da einfach zwischen Helm und meinem Kopf noch 'ne Schicht habe, weil ich ja nicht weiß, wer ist vorher damit gefahren." Es wird also ein zusätzliches Stück Schutzmaterial eingefügt, um sich bei der Nutzung eines fremden Helms wohler zu fühlen. Zugleich wird aber auch diese Anpassung relativiert und das eigene Empfinden in Frage gestellt: „Aber echt ist ja auch Schwachsinn, man könnte sich überwinden oder cooler sein. Aber das ist auch eher Typsache." (ql-06 Pos.21) Darüber hinaus wird davon berichtet, sich im Laufe der Nutzung sowohl kleidungstechnisch als auch bezüglich der eigenen körperlichen Empfindung besser an widrige Wetterbedingungen angepasst zu haben. „inzwischen [bin ich] auch, wie soll ich sagen, härter im Nehmen [...]. Ich hab' auch klamottentechnisch aufgerüstet. Ich fahre inzwischen auch, wenn es leicht regnet oder wenn es kalt ist." (ql-09 Pos.13) Die Nutzungspraktik hat sich im Verlaufe modifiziert.

Des Weiteren wird wertgeschätzt, sich nicht körperlich anstrengen zu müssen. Dies hat auch die Anmeldungsentscheidung positiv beeinflusst (vgl. Adoptionsprozess). „Deswegen bin ich sehr froh, dass es [EMS] gibt, dass ich nicht mehr mit dem Fahrrad den Berg hochfahren muss, sondern mit [dem EMS] fahren kann." (ql-08 Pos. 7)

\section{RAUMANEIGNUNG (materielle Aneignung)}

Die Fahrt mit dem Roller steht für viele in direktem Zusammenhang zum Erleben der räumlichen Außenwelt (ql-07 Pos.48-49). Neue Raumwirklichkeiten werden produziert: „kreuz und quer [...] gefahren und das war für mich eine wahnsinnig schöne Art die Stadt mal oberirdisch kennenzulernen." (ql-02 Pos.14) Die Stadt wird mit dem Roller erkundet, wobei auch ganz gezielt neue Wege gesucht werden: „durchaus [...] bewusst einen Umweg fahren oder durch eine Ecke der Stadt zu fahren, die man noch nicht kennt und

20 Dies kann auch als ein Adoptionsrisiko während der Phase der Adoption identifiziert werden: Das Teilen des Helms kann auch als unangenehm empfunden werden und je nach Ausprägung bis zu einer Ablehnung des Systems führen. 
dann ein bisschen schaut und sich auch verleiten lassen" (ql10 Pos.12, 15). Die Fahrt selbst mit dem Erlebnis des Raums und der Urbanität steht für sich - sie hat ihren einen eigenen Zweck, fern der zweckrationalen Distanzüberwindung. Teilweise führt dies auch zur Generierung weiterer Wege: Statt der kürzesten Strecke wird noch eine Runde gedreht bzw. die schönste Route gewählt (ql-01 Pos. 19).

\section{PRAKTISCHE ANEIGNUNG}

Die Häufigkeit der EMS-Nutzung in den ersten Monaten schwankt bei den interviewten Kund:innen. Während einige davon sprechen, dass sie sich erst an die Verfügbarkeit gewöhnen mussten (ql-11 Pos.49, ql-04 Pos.61), berichten andere darüber, dass sie gerade am Anfang besonders häufig gefahren sind (ql-06 Pos.46). Wieder andere erzählen, es habe sich über den Verlauf nicht viel verändert (ql05 Pos.36, ql-02 Pos.42). Auch stellt eine Person fest, dass sich der Nutzungszweck im Laufe der Erfahrungen verändert habe. Während zu Beginn die Buchung eher praktisch begründet war (von A nach B), so wird der Roller jetzt auch mal spontan an einem „coolen Samstag“ für „eine Rollertour an der Isar entlang" verwendet „just-for-fun“ (ql-07 Pos.32). Die Nutzung ist damit durch einen immateriellen Nutzwert begründet und beruht nicht allein auf zweckrationalen Motiven (ql-07 Pos.5-6). ${ }^{21}$

Darüber hinaus wird berichtet, dass sie die anfängliche Wahrnehmung der Kosten im Verlauf der Aneignung verändert hat (ql-11 Pos.49, 51-52). Interessant ist dabei der Vergleich, der im Laufe der Nutzung zu anderen Sharing-Angeboten gezogen wird, bspw. wird das EMS in einem Fall direkt mit den Gebühren für E-Tretroller verglichen. Der dort wahrgenommene höhere Preis führt letztlich zu einem entspannteren Verhältnis bei den Fahrten mit dem EMS, die Preissensibilität hat sich relativiert (ql-11 Pos.49, 51-52). Oder aber es wird eine Bilanz im Vergleich zur Nutzung eines (öffentlichen) Automobils gezogen, die positiv ausfällt (ql-07 Pos.3). Das letzte Beispiel zeigt deutlich, dass soziale Praktiken miteinander in Konkurrenz stehen und jede (Mobilitäts-) Praktik immer im Gesamtsystem Mobilität betrachtet werden muss. So kann die EMS-Praktik natürlich auch zu einer Verdrängung von anderen bestehenden Praktiken führen. ${ }^{22}$ In einem Beispiel wurde auf das ÖPNV-Monats-Ticket im Sommer verzichtet (ql-08 Pos.35). In einem anderen Interview wird davon berichtet, dass EMS vor allem bei schwierigen Topographien eine gute Alternative zum Fahrrad bietet (ql-08 Pos. 7). Wieder in einem anderen Beispiel erlangte die SharingPraxis im Allgemeinen einen großen Stellenwert (inkl. EMS), weil bewusst das Auto abgeschafft wurde (qi-07 Pos.3). Geht es konkret um die Entscheidung ,heute Auto, Roller oder UBahn?', wird im Vergleich zum Auto das besondere Fahrge-

21 Ergebnis der quantitativen Befragung: 4\% sind Fahrten „des Fahrens wegen"

22 Ergebnis der quantitativen Befragung: vgl. Kapitel 2 zu Substitutionseffekten - es fand eine Substitution über alle Verkehrsmittel hinweg statt. fühl hervorgehoben: „Es wäre mal cool, wenn das Wetter toll ist, wenn wir mit einem Roller irgendwo zum See fahren, statt [uns] ins Auto reinzuhocken" (ql-07 Pos. 10). Oder aber die Roller dienen als Alternative, um die „stickige U-Bahn/SBahn“ zu vermeiden und die „letzten Sonnenstrahlen“ zu genießen (ql-05 Pos.6).

\section{SOZIALE ANEIGNUNG}

In einigen Beispielen zeigen sich deutlich die Dynamiken, die durch die EMS-Praktik im sozialen Umfeld ausgelöst werden. Die Roller sind Gesprächsthema in der Familie, unter Freund:innen und Kolleg:innen (ql-08 Pos.38). Aufgrund der relativen Neuheit der Technologie erhalten die Nutzenden teils auch die Rolle von Pionieren, die nach ihrer Meinung und den jeweiligen Nutzungserfahrungen gefragt werden. Auch kommt man schnell mit anderen Nutzer:innen in Kontakt: Man fühlt sich einer Gruppe zugehörig und grüßt sich gegenseitig. Beim Parken wird ein kurzes Gespräch geführt oder einer ebenfalls an der Ampel stehenden EMS-Fahrer:in ein Lächeln zugeworfen (ql-03 Pos.32, ql-09 Pos.22). Die Praktik selbst wird damit auch als "sehr kommunikativ" bezeichnet (ql-08 Pos.38) - eine Praktik, die ,Laune macht'.

\subsection{Die internalisierte EMS-Praktik}

Gemäß dem theoretischen Modell hat sich in der letzten Phase des MoKa die neue EMS-Praktik als fester Bestandteil der individuellen Mobilitätspraxis herausgebildet. Verdrängungserscheinungen zu anderen Mobilitätspraktiken sind zwar feststellbar, doch tendenziell erscheint die EMS-Praktik unter den Befragten eher als punktuelle Ergänzung und Erweiterung der individuellen Mobilitätspraxis (ql-04 Pos. 63). Im Sample erfolgt die Nutzung des Rollers tendenziell spontan, je nach Lust und Laune und weiteren Kontextbedingungen wie z. B. Wetter oder Gepäck (ql-06 Pos. 6). Die Herausbildung eines routinisierten Verkehrsmittelwahlverhaltens (als Teil der EMS Praktik) lässt sich im Interview-Sample damit nicht feststellen.

Wichtiger Faktor, der Teil der EMS-Praktik ist und im Alltag zur spontanen Nutzung motiviert, ist das Spaßelement und die Freude beim Fahren des Rollers. Zur Relativierung wird in den Interviews häufig auch ${ }^{23}$ darauf hingewiesen, dass Nutzende nur deshalb kein schlechtes Gewissen haben, weil EMS mit (lokal) emissionsfreien Strom betrieben werden. Für manche dient sie auch als Mittel der sozialen Selbstexpression, gemäß dem Motto, wer dieses Mobilitätsangebot nutzt, bewegt sich ökologisch fort.

Insgesamt bewerten die Interviewten das Angebot und die Art der Fortbewegung sehr positiv (ql-12 Pos. 25). In der persönlichen Bilanzierung wird auch das Wort „großartig“ ver-

23 Ergebnis der quantitativen Befragung: EMS wird von 87\% nur gelegentlich (höchstens ein bis drei Tage im Monat) genutzt, eine allumfassende Substitution findet nicht statt. 
wendet (ql-02 Pos.30, ql-10 Pos. 18). Manche bezeichnen sich auch als echten „Fan“ (ql-09 Pos. 8). „Also [EMS] ist viel mehr als eine Bewegung von A nach B." (ql-10 Pos.10). Diese Aussage wird von mehreren in ähnlicher Weise bestätigt (ql12 Pos.12).

\section{Diskussion und Fazit}

In dem vorliegenden Artikel wurde der qualitative Teil einer EMS-Studie aus einer überwiegend praxeologischen Perspektive betrachtet. Im Vordergrund standen relevante Einflussfaktoren und wesentliche Elemente, die die EMS-Praktik selbst und ihre Herausbildung charakterisieren. Diese Erkenntnisse liefern schließlich eine Grundlage, die neue Praktik in ihrer Ausprägung besser zu verstehen. Auch schaffen sie eine Basis, um sie jenseits dieses Artikels in den Kontext einer nachhaltigen Stadtentwicklung einordnen zu können. Nur wenn die Elemente bekannt sind, die eine Praktik charakterisieren, lassen sich auch Ansatzpunkte zur Förderung, Optimierung oder auch Modifizierung ableiten.

Wenngleich hier nur ein Ausschnitt dargestellt werden kann, wird in der Ergebnisbeschreibung die Mannigfaltigkeit der konstituierenden Komponenten deutlich, die schließlich die EMS-Praktik als Ganzes bilden. Damit zeigt sich auch die Vielfalt des Handlungsspielraums für die Planungspraxis. Die Ergebnisse veranschaulichen das dynamische Wechselverhältnis in dem Individuum und Technik stehen. Erst bei der eigentlichen Ausübung einer Praktik und der subjektiven Wahrnehmung entfaltet eine Technologie ihren vollen Wert. Das Erleben der Fahrt mit allen Sinnen stellt ein zentrales Moment dar, die Emotionen werden direkt angesprochen. Für viele steht die Rollerfahrt auf einer symbolischen Ebene für Spaß und Freiheit. Die Daten bestätigen insgesamt die vielschichtige Bedeutung symbolischer und sozialer Faktoren im Umgang und mit der Nutzung von (Mobilitäts-) Technologien. Für die Entwicklung von Kommunikations- und Marketingstrategien bietet dies wichtige Ansatzpunkte.

Darüber hinaus wurde im Kontext der praktischen Aneignung festgestellt, dass die EMS-Praktik in den meisten Fällen niemals vollständig eine andere bestehende Praktik verdrängt. Das Dienstleistungsangebot wird eher in die individuellen Handlungsoptionen der Fortbewegung integriert. In der Regel wird damit die bereits vorhandene multimodale Mobilitätspraxis erweitert und gestärkt. Diese qualitativen Ergebnisse spiegeln sich auch in den quantitativen Daten der Studie wider.

Die neue Mobilitätsoption wird von den Interviewten sehr positiv bewertet. Die sehr positiven Einstellungen müssen jedoch relativiert werden. Vermutlich fand hier ein Selbstselektionseffekt statt, da v. a. gegenüber einem Produkt oder Thema positiv gestimmte Personen offener dafür sind, über ihre Erfahrungen damit zu berichten. Nichtsdestotrotz bie- ten die Daten eine sehr gute Grundlage, um die relevanten Perspektiven auf das Thema sichtbar zu machen. Diese können als Hypothesen in weiterführenden Studien abstrahiert und auch statistisch überprüft werden - zumindest in den Themenbereichen, in denen das Aufstellen quantifizierbarer Kategorien möglich und sinnvoll ist.

Für die Analyse der Interviewdaten hat sich die Orientierung an den zwei Modellen des MiMP und MoKa als sehr hilfreich erwiesen. Hier bietet sich noch viel Spielraum, die EMS-Praktik in all ihren Facetten noch tiefergehender zu analysieren. Eine Herausforderung bei Bezugnahme zu praxistheoretischen Ansätzen stellt jedoch das dahinterliegende philosophische Verständnis dar inklusive der Verwendung wenig alltagspraktischer Begrifflichkeiten. Im vorliegenden Artikel wurde eine möglichst hohe Vereinfachung angestrebt - eine damit einhergehende Reduzierung der theoretischen Differenziertheit und Verankerung wurde in Kauf genommen.

Im Diskurs zu einer zukunftsorientierten Stadtentwicklung spielen die Potenziale alternativer neuer Mobilitätsangebote eine wesentliche Rolle. EMS bildet einen Baustein im Mobilitätsystem einer Stadt, der das multimodale Angebot insgesamt attraktiver machen kann. Wie in den Daten sichtbar wurde, werden die Roller vor allem auch genutzt, um Nachteile bei bestehenden anderen Mobilitätsoptionen auszugleichen. Für die hier interviewten Personen bietet das System also einen Mehrwert, der nicht durch andere Optionen ersetzt werden kann, der aber auch kein anderes Angebot vollständig substituiert. Neben der zweckrationalen Raumüberwindung bedeutet das Fahren mit dem Roller aber vor allem auch Spaß - die Praktik des EMS ist schließlich mehr als nur reine Fortbewegung.

\section{Literaturverzeichnis}

Aguilera-García, Álvaro, Juan Gomez, und Natalia Sobrino. 2019. Exploring the adoption of moped scooter-sharing systems in Spanish urban areas. Cities 96: 1-13.

Aguilera-García, Álvaro, Juan Gomez, Natalia Sobrino, und Juan José Vinagre Díaz. 2021. Moped Scooter Sharing: Citizens' Perceptions, Users' Behavior, and Implications for Urban Mobility. Sustainability 13: 6886.

Degele, Jutta et al. 2018. Identifying E-Scooter Sharing Customer Segments Using Clustering. In 2018 IEEE International Conference on Engineering, Technology and Innovation (ICE/ITMC), 1-8. Stuttgart: IEEE https://ieeexplore.ieee. org/document/8436288/. Zugegriffen: 22. November 2019.

Degele, Nina. 2002. Einführung in die Techniksoziologie. München. 
Le Bris, Jessica. 2015. Die individuelle Mobilitätspraxis und Mobilitätskarrieren von Pedelec-Besitzern. Adoption und Appropriation von Elektrofahrrädern. Dissertation, Tübingen: Universität Tübingen https://publikationen.uni-tuebingen.de/xmlui/handle/10900/71968.

Mey, Günter, und Katja Mruck. 2011. Grounded-TheoryMethodologie: Entwicklung, Stand, Perspektiven. In Grounded Theory Reader, 11-48. Wiesbaden: VS Verlag für Sozialwissenschaften.

Oudshoorn, Nelly EJ, und Trevor Pinch. 2007. User-technology relationships: Some recent developments. In Handbook for Social Studies of Science, 541-567. MIT press.

Pérez-Fernández, Onel, und Juan Carlos García-Palomares. 2021. Parking Places to Moped-Style Scooter Sharing Services Using GIS Location-Allocation Models and GPS Data. ISPRS International Journal of Geo-Information 10: 230.

Pinch, Trevor J., und Wiebe E. Bijker. 1984. The Social Construction of Facts and Artefacts: or How the Sociology of Science and the Sociology of Technology might Benefit Each Other. Social Studies of Science 14: 388-441.

Rogers, Everett. 2003. Diffusion of Innovations. 5th Edition.

Shove, Elizabeth, Mika Pantzar, und Matt Watson. 2012. The Dynamics of Social Practice: Everyday Life and how it Changes. London: SAGE Publications Ltd.

Silverstone, Roger, und Eric Hirsch. 1992. Consuming Technologies: Media and information in domestic spaces. Taylor \& Francis Ltd.

\section{Autor:innenangaben}

\section{Dr. Jessica Le Bris}

Green City Experience GmbH

Türkenstrasse 55-57

80799 München

Deutschland

E-Mail: jessica.le-bris@greencity.de

\section{Julia Korsten}

Green City Experience $\mathrm{GmbH}$

Türkenstrasse 55-57

80799 München

Deutschland

E-Mail: julia.korsten@greencity.de

\section{Verena Prediger}

Green City Experience $\mathrm{GmbH}$

Türkenstrasse 55-57

80799 München

Deutschland

E-Mail: verena.prediger@greencity.de

\section{Dr. Alexandra Bensler}

\author{
Green City Experience GmbH \\ Türkenstrasse 55-57 \\ 80799 München \\ Deutschland
}

E-Mail: alexandra.bensler@uni-bremen.de 